Pacific Journal of Mathematics

HOMOLOGICAL ALGEBRA OF STABLE HOMOTOPY RING $\pi$
OF SPHERES 


\title{
HOMOLOGICAL ALGEBRA OF STABLE HOMOTOPY RING $\pi_{*}$ OF SPHERES
}

\author{
T. Y. LIN
}

The stable homotopy groups are studied as a graded ring $\pi_{*}$ via homological algebra. The main object is to show that the projective (and weak) dimension of a finite type $\pi_{*}$-module is $\infty$ unless the module is free. As a corollary, a partial answer to Whithead's corollary to Freyd's generating hypothesis is obtained.

1. Introduction and statement of main results. It is wellknown that the stable homotopy groups of spheres form a commutative graded ring $\pi_{*}$ [20]. This paper is our first effort towards the investigation of the homological properties of the stable homotopy ring $\pi_{*}$. In this paper we have completed the computations of all the homological numerical invariants of finitely generated type. The nonfinitely generated type will be taken up in forth-coming papers.

The paper is organized as follows: The introduction is $\S 1$. In $\$ 2$ we give a brief exposition about the theory of graded rings, which are needed in later sections. $\S 3$ is primarily a preparation for $\S 4$. Here the finitistic global dimensions of the " $p$-primary component" $\Lambda_{p}$ of $\pi_{*}$ (precisely, $\Lambda_{p}$ is a ring obtained by localizing $\pi_{*}$ at a maximal ideal) are computed, and a geometric realization of $\Lambda_{p}$ is constructed. $\S 4$ is the mainbody of this paper, here we prove Theorem 2 and derive from it Theorems 1, 3, and 4. We would like to suggest that the reader, after $\S 1$, go directly to $\S 4$ and refer to the rest of the sections when necessary.

The author would like to thank Professor W. Massey for his encouragement and fruitful discussions.

The main results are:

THEOREM 1. Let $A$ be a locally finitely generated (i.e., there are only finitely many generators at each degree) $\pi_{*}$-module having finite projective, as well as weak, dimension. Then $A$ is a free $\pi_{*}$-module and hence is realizable as a stable homotopy module $\pi_{*}(Y)$ by a wedge $Y$ of spheres.

In [7] Freyd propose a conjecture, known as the generating hypothesis, which asserts that a map between finite $C W$-complexes, which induces the zero map on stable homotopy groups, is stably null-homotopic. A consequence of this conecture, due to G. Whithead, asserts that the finitely generated stable homotopy module $\pi_{*}(X)$ of 
a finite specturum is a stable homotopy module of a wedge of spheres. Theorem 1 implies that a finitely generated stable homotopy module of finite projective dimension is a stable homotopy module of a wedge of spheres. Thus Theopem 1 proves "Whithead's corollary to Freyd's generating hypothesis" with one additional condition.

THEOREM 2. Let $Y$ be a connected spectrum having a finite skeleton in each dimension. Then the projective, as well as weak, dimension of $\pi_{*}(Y)$ as a $\pi_{*}$-module is infinite unless $Y$ is a wedge of spheres.

In the notation of $\S 2,3$ below, we have.

$$
\begin{aligned}
\text { Theorem 3. ( i) } & f \cdot P \cdot D\left(\pi_{*}\right)=f \ell \cdot P \cdot D\left(\pi_{*}\right)=0<F \cdot P \cdot D\left(\pi_{*}\right) \neq 0 \\
& \text { (ii) } f \cdot W \cdot D\left(\pi_{*}\right)=f \ell \cdot W \cdot D\left(\pi_{*}\right)=0 \leqq F \cdot W \cdot D\left(\pi_{*}\right) .
\end{aligned}
$$

It is well known that the projective dimension of a module over the Steenrod Algebra $(\bmod p)$ is either 0 or $\infty$. Theorem 3 implies that an analogous situation also occurs in the stable homotopy ring, when a finiteness condition is imposed. For $f \iota . P . D\left(\pi^{*}\right)=0$ means that the projective dimension of a locally finitely generated $\pi_{*}$-module is either 0 or $\infty$. Note that the finiteness condition cannot be removed. For $F . P . D\left(\pi_{*}\right)>0$ implies that there are some $\pi_{*}$-modules having projective dimension $\geqq 1$. In fact, in [14] we show that the only projective dimension of $\pi_{*}$-module is either 0,1 , or $\infty$, equivalently $F \cdot P . D\left(\pi_{*}\right)=1$.

TheOREm 6. The projective, as well as weak, dimension of an ideal in $\pi_{*}$ is infinite.

This theorem shows that $\pi_{*}$ is a very nonhereditary ring.

Since we are working with the stable theory, a good category will be helpful. Therefore we will freely use Boardman's stable category of (pointed) $C W$-spectra. [6].

\section{Convention:}

(i) All the spectra $X$ are assumed to be connected, i.e., there is some integer $n_{0}$ such that $\pi_{n}(X)=0$ for $n<n_{0}$.

(ii) All the modules are assumed to be bounded from below, i.e. there is some integer $n_{0}$ such that $A_{n}=0$ for all $n<n_{0}$; see $\S 2.3$.

(iii) We will not distinguish between a map or its homotopy class and a spectrum or its homotopy type.

2. Graded rings. The purpose of this section is to give a brief 
exposition about the theory of graded rings, which are needed in later sections. First, we define a graded ring and set up the category of its modules; also we recall here a few terms and facts about homological algebra. Secondly, we briefly discuss a graded version of the theory of Noetherian rings: Since the finitely generated condition is too strong for homotopy [10], we relax the finiteness condition and arrive at a generalization-called a locally Noetherian ring [16]. Then we prove some folk theorems about such rings. These theorems are standard in the ordinary Noetherian theory of rings, yet the graded versions seem never to have appeared in print. Thirdly, we review some facts about the localization. Finally we introduce an operation on graded modules - the lifting of a module. This operation gives us a clearer view of graded free modules, and implies clearly the realizability of free $\pi_{*}$-modules and mappings between them.

\section{First let us recall some definitions.}

Definitions 2.1. A graded ring $R$ is a sequence of abelian groups $R_{n}$ for all NONNEGATIVE integers $n \geqq 0$ together with homomorphisms $R_{n} \otimes_{z} R_{m} \rightarrow R_{n+m}$ (which we call multiplication) satisfying associativity and distributivity. We always assume the existence of a multiplicative identity element 1 in $R_{0}$.

A graded left $R$-module $A$ is a sequence of abelian groups $A_{n}$ for $A L L$ integers $n$, together with homomorphisms $R_{n} \otimes_{z} A_{m} \rightarrow A_{n+m}$ (which we call scalar multiplication) satisfying the usual module identities.

A graded ring is said to be commutative (in the graded sense) if $\alpha \cdot \beta=(-1)^{n \cdot m} \beta \cdot \alpha$ for $\alpha \in R_{n}$ and $\beta \in R_{m}$. The integer $n$ will be called the degree of $\alpha$ and denoted by $\operatorname{deg} \alpha$.

From now on, all rings will be assumed to be commutative (in the graded sense); all modules, ideals and properties will be assumed to be two sided unless they are specifically called otherwise. All modules, rings and ideals are graded.

An $R$-module map $f: A \rightarrow B$ of degree $d$ is a sequence of maps $f: A_{n} \rightarrow B_{n \div d}$ such that for $\alpha \in R_{n}$ and $x \in A_{m} f(\alpha x)=(-1)^{n d} \alpha f(x)$.

Topological Examples. 2.2. Let $\pi_{n}=\pi_{n}\left(S^{0}\right)$ be the $n$th stable homotopy group of the 0 -sphere spectrum. Then the collection $\pi_{*}=$ $\left\{\pi_{n}, n \geqq 0\right\}$, with composition of maps as multiplication, is a graded commutative ring [20]. 
Let $X$ be a spectrum, then the collection of stable homotopy groups $\pi_{*}(X)=\left\{\pi_{n}(X),-\infty<n<\infty\right\}$ is a graded $\pi_{*}$-module with composition of maps as scalar multiplication. Similarly a stable cohomotopy group $\pi^{*}(X)=\left\{\pi^{n}(X),-\infty<n<\infty\right\}$ is a graded left $\pi_{*}$-module. Moreover, since $\pi_{*}$ is commutative, a right $\pi_{*}$-module can be changed into a left $\pi_{*}$-module by standard sign convention. Thus throughout the whole paper we will omit the term left or right, and leave the reader to interpret it properly.

Next we will recall some notions from homological algebra.

Definitions. 2.3. Let $\mathscr{C}(R)$ be the category of $R$-modules bounded from below; that is, (i) the objects are the $R$-modules $A=\left\{A_{n} ;-\infty<n<\infty\right\}$ such that there is an integer $n_{6}$ with $A_{n}=0$ for all $n<n_{0}$, and (ii) the morphisms are $R$-module maps of degree 0 . Let $P . d_{R} A$ and $W . d_{R} A$ be the projective and weak dimensions, respectively, of the $R$-medule $A$ in the category $\mathscr{C}(R)$ (Note that the notions of projective and weak dimension are categorical in nature, so it is important to require that everything is INSIDE THE CATEGORY $\dddot{l l}(R)$ ). Following [3, pp. 478] or [9, pp. 48] we write

$$
\begin{aligned}
G . D(R) & =\operatorname{Sup}\left\{P \cdot d_{R} A \mid A \in \mathscr{K}(R)\right\} \\
F . P . D(R) & =\operatorname{Sup}\left\{P \cdot d_{R} A \mid A \in \mathscr{C l}(R), P . d_{R} A<\infty\right\} \\
f \ell . P . D(R) & =\operatorname{Sup}\left\{P . d_{R} A \mid A \in \mathscr{C l}(R), P . d_{R} A<\infty\right.
\end{aligned}
$$

and $A$ is locally finitely generated\}

$$
f . P . D(R)=\operatorname{Sup}\left\{P . d_{R} A \mid A \in \mathbb{C}(R), P . d_{R} A<\infty\right.
$$

and $A$ is finitely generated\}, where "Sup" stands for the supremum, and "locally finitely generated" means that there is a set of generators which consists of only FINITEL Y MANY generators EACH DEGREE. (see 2.6 below). Similarly we can define $F \cdot W \cdot D(R), f \ell W \cdot D(R)$ and $f . W . D(R)$ by replacing $P . d_{R} A$ with $W . d_{R} A$. (The notations G.D, F.P.D, f.P.D are standard, while $f \ell . P . D$ is introduced here; the " $f \ell$ " stands for "finite dimension and locally finitely generated").

\section{Let us recall}

Proposition 2.4. Let $0 \rightarrow A^{\prime} \rightarrow A \rightarrow A^{\prime \prime} \rightarrow 0$ be a short exact sequence of $R$-modules. Then if $A$ is projective and $A^{\prime \prime}$ is not, $P . d_{R} A^{\prime}=P . d_{R} A^{\prime \prime}-1$.

Corollary 2.5. F.P.D $(R) \neq 0$ (or $f \ell \cdot P \cdot D(R) \neq 0$ ) implies that there is a module $A$ with $P . d_{R} A=1$.

Proof. By definition F.P. $D(R) \neq 0$ (or $f \iota \cdot P \cdot D(R) \neq 0$ ) implies that 
there is a module, say $B$, such that $P \cdot d_{R} B=n<\infty$. Let

$$
0 \leftarrow B \leftarrow R_{0} \leftarrow P_{l} \leftarrow \cdots \leftarrow P_{n} \leftarrow 0
$$

be a projective resolution. Then we can break the resolution into short exact sequences

$$
\begin{gathered}
0 \leftarrow B \leftarrow P_{0} \leftarrow K_{0} \leftarrow 0 \\
0 \leftarrow K_{i} \leftarrow P_{i+1} \leftarrow K_{i+1} \leftarrow 0,0 \leqq i \leqq n-2 \\
0 \leftarrow K_{n-2} \leftarrow P_{n-1} \leftarrow p_{n} \leftarrow 0 .
\end{gathered}
$$

By Proposition 2.4 we get $P . d_{R} K_{0}=1$. Taking $A=K_{0}$ finishes the proof.

II. We also need a graded version of Noetherian rings.

Definitions. 2.6. An $R$-module $A$ is said to be locally finitely generated if there is a set $X$ of generators such that (i) $X \cap A_{n}$ is a finite set and (ii) there exists an integer $n_{0}$ such that $X \cap A_{m}=$ empty set for $m<n_{0}$. (Note that if $A \in \mathscr{l l}(R)$, i.e., $A$ is bounded from below, then condition (ii) is automatically satisfied)

An $R$-module $A$ is said to be locally Noetherian if every submodule is locally finitely generated. A ring is locally Noetherian if it is locally Noetherian as a module.

There is a simple criterion for locally Noetherianness

Proposition 2.7. $R$ is locally Noetherian if and only if $R_{0}$ is an ungraded Noetherian ring and each $R_{n}$ is a finitely generated $R_{0}$-module.

Proof. To prove necessity, let $I_{0}$ be an ideal of $R_{0}$. Then $I=$ $I_{0} \cup\left(\cup_{n \geqq 1} R_{n}\right)$ is an ideal of $R$ and hence, by assumption, is locally finitely generated; therefore $I_{0}$ and each $R_{n}, n \geqq 1$ are finitely generated. Thus we establish the necessity. Sufficiency holds because if $I$ is an ideal of $R$, then, by locally Noetherianness, each $I_{n}$ is a finitely generated $R_{0}$-module.

Topological example 2.8. The stable homotopy ring $\pi_{*}$ is a locally Noetherian ring (by Serre's theorem and 2.7). The stable homotopy module is locally Noetherian if $X$ is a connected spectrum having a finite skeleton in each dimension. (see 2.9 (i) below).

Most of the (ordinary) Noetherian theorems can easily be rephrased to get valid graded versions. The following proposition is a graded 
version of a standard Noetherian theorem.

Proposition 2.9. (i) A locally finitely generated module over a locally Noetherian ring is locally Noetherian. (ii) A locally finitely generated flat module of a locally Noetherian ring is projective.

The author learned this proposition from $P$. May in a course on homological algebra [16]. Since the proposition seems not to appear in print, we reproduce the proof here.

Proof. (i) The proof is almost the same as in the ungraded case. First let us note that a quotient of a locally Noetherian module is locally Noetherian; also let us observe that a locally finitely generated free module of a locally Noetherian ring is locally Noetherian. Then, since every locally finitely generated $R$-module $A$ is a quotient of a locally finitely generated free $R$-module, so the module $A$ is locally Noetherian.

(ii) Let $A$ be a locally finitely generated $R$-module, then there is a locally finitely generated free $R$-module $F$ such that

$$
0 \rightarrow K \rightarrow F \rightarrow A \rightarrow 0
$$

is exact. Since $R$ is locally Noetherian, so by (i) $F$ is locally Noetherian; hence $K$ is locally finitely generated. Thus, in order to prove that $A$ is projective, it is sufficient to prove the following Lemma.

Lemma 2.10. Let $0 \rightarrow K \stackrel{\psi}{\rightarrow} F \rightarrow A \rightarrow 0$ be a short exact sequence of $R$-modules, where $A$ is flat, $F$ is free and $K$ is locally finitely generated. Then the sequence splits, hence $A$ is projective.

Proof. Without loss of generality, we can assume, since $A, F$ and $K$ are bounded from below, that $A, F$ and $K$ have no negative degrees (i.e. $F_{i}=K_{i}=A_{i}=0$ for $i<0$ ). We will prove this lemma by constructing $\phi: F \rightarrow K \ni \phi \psi=1$ : Let $F(q)$ be the submodule generated by $F_{i}, i \leqq q$ and define $K(q)$ similary. Observe that $\psi(K(q)) \subset F(q)$. We will define $\phi(q): F(q) \rightarrow K(q)$ inductively to satisfy $\phi(q) \psi=1$ on $K(q)$. We start with $\phi(-1)=0$; we suppose that $n \geqq 0$ and $\phi(n-1)$ has been defined. In order to construct $\phi(n)$, first we remark that if $x \in K_{n}$, then there exists a map $\lambda: F(n) \rightarrow K(n)$ such that (i) $\lambda \psi=1$ on $K(n-1)+R x$ and (ii) $\lambda=$ $\phi(n-1)$ on $F(n-1)$. To prove this remark, let $\left\{y_{j}\right\}$ be a basis for $F$ and suppose that $\psi(x)=\sum_{h} \gamma_{j_{h}} y_{j_{h}}$, where $\gamma_{j_{h}} \in R$ and $y_{j_{h}} \in\left\{y_{j}\right\}$. Let $I$ be the left ideal generated by $\left\{\gamma_{j_{h}}\right\}$. Then, since $A$ is flat, we 
have $I F \cap K=I K$ (see the Sublemma 2.11 below). So $x=\sum \gamma_{j h} \cdot k_{j h}$ for some $k_{j_{h}} \in K$. If $\gamma_{j_{h}} \cdot k \in K(n-1)$, then $\gamma_{j_{h}} \cdot k_{j_{h}}=\dot{\phi}(n-1) \psi\left(\gamma_{j h} k_{j h}\right)$. Define $\lambda$ by

$$
\lambda=\phi(n-1) \text { on } F(n-1) ;
$$

$\lambda\left(y_{j_{h}}\right)=k_{j_{h}}$, if $y_{j_{h}} \in F_{n}, \gamma_{j_{h}} \in R_{0}$ and $\gamma_{j_{h}} \neq 0$; and $\lambda\left(y_{j}\right)=0$, if $y_{j} \in F_{n}$ and dose not appear in $\left\{y_{j_{h}}\right\}$. Then $\lambda \psi^{\prime}(x)=x$, and this proves our remark.

Proceeding inductively, suppose that $\lambda_{\ell-1}: F(n) \rightarrow K(n)$ can be constructed so as to satisfy

$$
\begin{aligned}
\lambda_{\ell-1} & =\phi(n-1) \text { on } F(n-1) ; \text { and for any } x_{1}, \cdots, x_{\ell-1} \in K_{n}, \\
\lambda_{\ell-1} \cdot \gamma^{\prime} & =1 \text { on } K(n-1)+R x_{1}+\cdots+R x_{\ell-1} .
\end{aligned}
$$

Suppose given $x_{1}, x_{2}, \cdots, x_{\ell} \in K_{n}$. Construct $\lambda$ as above for $K(n-1)+$ $R x_{\ell}$ and construct $\lambda_{\ell-1}$, as assumed possible, for $K(n-1)+R w_{1}+\cdots+$ $R w_{\ell-1}$, where $w_{i}=x_{i}-\lambda \psi\left(x_{i}\right)$. Now define $\lambda_{\ell}=\lambda_{\ell-1}+\lambda-\lambda_{\ell-1} \psi \lambda$. Then

$$
\lambda_{\ell}=\phi(n-1) \text { on } F(n-1) \text {; }
$$

and

$$
\lambda_{\ell} \psi\left(x_{i}\right)=x_{i} \quad 0 \leqq i \leqq \ell
$$

Since $K$ is locally finitely generated, it follows that we can construct $\phi(n)$. Since $F=\cup_{n} F(n)$, we can find $\phi: F \rightarrow K$ such that $\phi \dot{\psi}=1$. This completes the Lemma.

The only thing left is to show

Sublemma 2.11. The following are equivalent

(i) $A$ is a flat $R$-module

(ii) For every ideal $I$ of $R$, and for every short exact sequence $0 \rightarrow K \rightarrow F \rightarrow A \rightarrow 0$ with $F$ free, we have $I F \cap K=I K$.

Proof. Consider the following commutative diagram

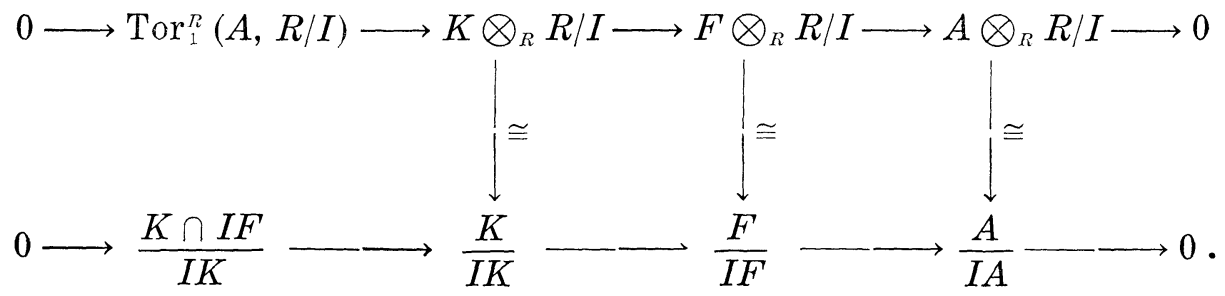

Note that the top horizontal line is exact since it is a part of a long exact sequence of the functor Tor; and the bottom line is easily 
seen to be exact. The right three vertical arrows are the natural isomorphisms induced by scalar multiplication. Hence

$$
\frac{K \cap I F}{I K} \cong \operatorname{Tor}_{1}^{R}(A, R / I) .
$$

Thus

$$
\frac{K \cap I F}{I K} \cong \operatorname{Tor}_{1}^{R}(A, R / I)=0
$$

if and only if $A$ is flat. Or equivalently, $K \cap I F=I K$ if and only if $A$ is flat.

The next propositions are also standard in Noetherian theory (though the ring need not be locally Noetherian, the locally finiteness conditions are essential).

Proposition 2.12. (graded Nakayama Lemma)

The following conditions on an ideal $I$ in $R$ are equivalent

(i) $I \subset J=$ the Jacobson radical of $R$.

(ii) Let $A$ be a locally finitely generated $R$-module and $H$ be a submodule of $A$. Then $H+I A=A$ implies that $H=A$.

(iii) Let $A$ be a locally finitely generated $R$-module. Then $I A=$ $A$ implies that $A=0$.

Proof. Before we start to prove the equivalence, let us first observe that every proper submodule $H$ of a locally finitely generated $R$-module $A$ is always contained in a proper maximal submodule: Suppose $H \neq A=\left\{A_{n} ;-\infty<n<\infty\right\}$. Then we will construct a proper maximal submodule $G \ni H \subset G \varsubsetneqq A$. Let $m-1$ be the highest degree such that $H_{m-1}=A_{m-1}$. Since $A$ is locally finitely generated, $A_{m}$ is finitely generated over $R_{0}$ (= the 0 th component of $R$ ). Then, by Zorn's Lemma, we can find a maximal proper $R_{0}$-submodule $E$ (of $A_{m}$ ) containing $H_{m}$. Let $G$ be an $R$-submodule defined by

$$
G_{n}= \begin{cases}A_{n}, & \text { if } n \neq m \\ E, & \text { if } n=n .\end{cases}
$$

It is not difficult to see that $G$ is, indeed, an $R$-submodule (Let $H^{(m-1)}$ be the subset consisting of all $H^{n}, n \leqq m-1$. Then, by construction, $H^{(m-1)}=G^{(m-1)}=A^{(m-1)}$. Since $R \cdot H^{(m-1)} \subset H$, we have $R \cdot G^{(m-1)} \subset H \subset G$. Therefore $R \cdot G \subset G$. Thus $G$ is, indeed, a submodule). The maximality of $G$ (as $R$-submodule) follows immediately from the maximality of $E$ as $R_{0}$-submodule of $A_{n}$.

Now with this observation, we are ready for proving the 
equivalences.

(i) $\Rightarrow$ (ii). First we note that $J \cdot A \subset J(A)=$ the intersection of all maximal submodules (Since $J(A)$ is the set of elements of $A$ belonging to the kernels of all homomorphisms of $A$ into simple $R$-modules, and $J$ annihilates all simple $R$-modules). Next let us assume, to the contrary, that $H \neq A$. Since $A$ is locally finitely generated, by the previous remarks, there is a proper maximal $R$-submodule $G$ containing $H$. Since $I \cdot A \subset J \cdot A \subset J(A)$, we have $I \cdot A \subset G$. Therefore $I A+H \subset G \neq A$; contradiction. This establishes the implication (i) $\Rightarrow$ (ii)

(ii) $\Rightarrow$ (iii). Take $H=0$ in (ii); we get the conclusion.

(iii) $\Rightarrow$ (i). If $B$ is a maximal ideal, then, by (iii), $I \cdot(R / B)=0$. In other words, $B \supset I \cdot R=I$. Since $B$ is arbitrary, $I \subset J=$ the Jacobson radical of $R=$ the intersection of all the maximal ideals.

Proposition 2.13. Let $C(R) \subseteq \mathscr{l}(R)$ be the full subcategory of locally finitely generated projective $R$-modules. Let $I$ be an ideal of $R$ contained in the Jacobson radical of $R$. Set $R^{\prime}=R / I$ and write $A^{\prime}=A \bigotimes_{R} R^{\prime}=A / I A$ for $A \in \mathscr{M}(R)$. Then

$$
\text { ': } C(R) \longrightarrow C\left(R^{\prime}\right)
$$

is a full additive functor satisfying the following property: If $f: P \rightarrow Q$ is a morphism in $C(R)$ such that $f^{\prime}: P^{\prime} \rightarrow Q^{\prime}$ is an isomorphism in $C\left(R^{\prime}\right)$, then $f$ is an isomorphism. (This proposition is the graded version of [5, proposition 2.12, p. 90 ] and the proof is essentitally the same.)

Proof. Given $f^{\prime}: P^{\prime} \rightarrow Q^{\prime}$ there is an $f: P \rightarrow Q$ making the diagram

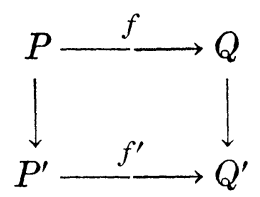

commute. This is because $P$ is projective and $Q \rightarrow Q^{\prime}$ is onto. Thus the functor ' $'$ is full. If $f^{\prime}$ is onto, then it follows from the Nakayama Lemma 2.12 (ii) (since $Q$ is locally finitely generated) that $f$ is onto. The projectivity of $Q$ now implies that $f$ is a spilit epimorphism. It follows that $H=\operatorname{Ker} f$, being a direct summand of $P$, is locally finitely generated. Now since $\operatorname{Ker} f^{\prime}=0, H^{\prime}=0$; or equivalently $H / I \cdot H=0$. By the Nakayama Lemma 2.12 (iii), we get $H=0$. This proves the proposition.

CoRollary 2.14. (i) A locally finitely generated projective (or 
flat) $\pi_{*}$-module $A$ is free.

(ii) A locally finitely generated projective (or flat) module $A$ over a local ring (= locally Noetherian ring with unique maximal ideal) is free.

Proof. (i) Observe that the Jacobson radical of $\pi_{*}$ is $\pi_{+}=$ $\left\{\pi_{n}, n>0\right\}$. Take $I=\pi_{+}$as above. Then $\pi_{*}^{\prime}=\pi_{*} / \pi_{+} \cong \pi_{0}$ is isomorphic to the $\operatorname{ring} Z$ of integers, so $A^{\prime} \in C(Z)$ is free. By Proposition $2.13, A$ is free.

(ii) Take $I=$ the Jacobson radical (= the unique maximal ideal) above. Then $R^{\prime}$ is field, so $A^{\prime}$ is free. By Proposition 2.13, $A$ is free.

Theorem 2.15. (i) If $A \in \mathscr{l}(R)$ and $P . d_{R} A=n<\infty$, then there is a free resolution of length $n$, i.e., the sequence

$$
0 \longleftarrow A \longleftarrow F_{0} \longleftarrow F_{1} \longleftarrow \cdots \longleftarrow F_{n} \longleftarrow 0
$$

is exact and each $F_{i}$ is free.

(ii) Let $R$ be the stable homotopy ring $\pi_{*}$ or an arbitrary local ring; and let $A$ be a locally finitely generated module. Then P. $d_{R} A=$ $n<\infty$ implies that there is a locally finitely generated free resolution of length $n$, i.e., the sequence

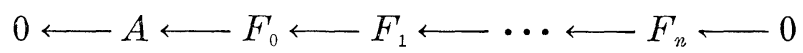

is exact and each $F_{i}$ is a locally fnitely generated free $R$-module.

Proof. (i) By assumption P. $d_{R} A=n<\infty$, there is an exact sequence

$$
0 \longleftarrow A \longleftarrow F_{0} \longleftarrow F_{1} \longleftarrow \ldots \stackrel{d_{n-1}}{\longleftarrow} F_{n-1} \stackrel{d_{n}}{\longleftarrow} K_{n} \longleftarrow 0
$$

such that $F_{i}$ are free and $K_{n}$ is projective. By the Eilenberg Lemma [4, pp. 24], there is a very large free module, say $F$, such that $K_{n} \oplus F$ is a free module. Then the following sequence

$$
0 \longleftarrow A \longleftarrow F_{0} \longleftarrow \cdots \stackrel{d^{\prime} n-1}{\longleftarrow}\left(F_{n-1} \oplus F\right) \stackrel{d_{n}^{\prime}}{\longleftarrow}\left(K_{n} \oplus F\right) \longleftarrow 0
$$

is a free resolution for $A$, where $d_{n}^{\prime}=d_{n} \oplus 1_{F} \quad\left(1_{F}\right.$ is the identity map on $F), d_{n-1}^{\prime}=d_{n-1} \oplus 0$ ( 0 is the zero map $\left.F \rightarrow F_{n-2}\right)$; and $d_{i}^{\prime}=d_{i}$, $0 \leqq i \leqq n-2$. This proves (i).

(ii) By Corollary 2.14, any projective resolution is a free resolution. The proof that the resolution is locally finitely generated is similar to the ordinary Noetherian case.

III. The localization can also be carried over to the graded commutative ring $R$. (Note that since the ring $R$ is not strictly 
commutative, some special care has to be taken [18]). For simplicity we will assume that the elements of the multiplicative system $S$ (containing 1 but not containing 0 ) are of degree 0 , i.e. $S \subset R_{0}$. Let $R \otimes S$ be the Cartesian product, then we will write

$$
(a, s) \sim\left(a^{\prime}, s^{\prime}\right) \text { if } \quad \exists t \in S \ni t \cdot\left(a s^{\prime}-a^{\prime} s\right)=0
$$

where $(a, s)$ and $\left(a^{\prime}, s^{\prime}\right)$ are elements of $R \times S$. It is easy to verify that this is, in fact, an equivalence relation. Let the equivalence class to which $(a, s)$ belongs be denoted by $a / s$ and be assigned a degree, $\operatorname{deg} a / s=\operatorname{deg} a$. We will denote by $R_{S}$ the totality of the equivalence classes. As in the ordinary case, we can make $R_{S}$ into a ring by introducing the sum and product with the usual formulas. Moreover there is a natural map

$$
R \longrightarrow R_{S}
$$

which sends $a$ to $a / 1$ (Note that this map, in general, is not a monomorphism). We will call this map a natural embedding (abuse of language). With this natural embedding we can turn $R_{S}$ into an $R$-module.

We also can consider the localization of a module. Let $A$ be an $R$-module, then the localized module is defined to be $A_{S}=A \otimes_{S} R_{S}$.

Proposition 2.16. If $A$ is a flat $R$-module, then $A_{S}$ is flat $R$-module, in particular $R_{S}$ is a flat $R$-module.

The proof of this proposition can be found in [17, pp. 170, Lemma 2].

\section{Finally we recall}

Definition 2.17. Let $A=\left\{A_{n},-\infty<n<\infty\right\} \in \mathscr{C l}(R)$. Let $L A=$ $\left\{(L A)_{n},-\infty<n<\infty\right\}$ to be the $R$-module such that (i) $(L A)_{n}=A_{n-1}$ as Abelian group and (ii) $\gamma \cdot L a=(-1)^{\operatorname{deg} \gamma} L \gamma a$, where $\gamma \in R$ and $a \in A$. Inductively we define $L_{\ell} A=L\left(L_{\ell-1} A\right)$.

It is easy to see that $L_{\ell} R$ is a free $R$-module with one single generator $L_{\ell} 1$ ( 1 is the identity in $R$ ) at degree $\ell$. Note also that a free $R$-module $F$ is a direct sum of the form $L_{\ell} R$ i.e., $F \cong \sum_{i} \oplus$ $\left(L_{\ell_{i}} R\right)$.

In the case $\pi_{*}$ is the ring, then $L_{\ell_{i}} \pi_{*}$ is just the stable homotopy module $\pi_{*}\left(S^{\ell_{i}}\right)$ of the $\ell_{i}$-sphere. Thus we have a theorem analogous to $[2, \S 3$, Theorem 3$]$, namely,

Theorem 2.18. Let $F \in \mathscr{C l}\left(\pi_{*}\right)$ be a locally finitely generated 
free $\pi_{*}$-module. Then there exists a wedge $N$ of spheres, which has only finitely many spheres at each dimension, such that

(i) $\pi_{*}(N) \cong F$ as $\pi_{*}$-module; and

(ii) $\pi_{0}(N, X) \cong \operatorname{hom}_{\pi_{*}}\left(F, \pi_{*}(X)\right)$ for any specturum $X$, where $\pi_{0}(N, X)$ is the homotopy classes of maps from $N$ to $X$, and hom ${ }_{\pi^{*}}$ $\left(F, \pi_{*}(X)\right)$ is the homomorphism of degree 0 from $F$ to $\pi_{*}(X)$.

3. The p-primary component of $\pi_{*}$. Identify $\pi_{0}$ with ring $Z$ of integers. Let $M_{p}$ be the maximal ideal of $\pi_{*}$ generated by the prime $p$ and $\pi_{+}$, where $\pi_{+}$are the elements of degree $\geqq 1$. The complement of $M_{p}$ is obviously a multiplicative system of degree 0 ; hence, by $\S 1$. III, upon localizing at this multiplicative system we get a local ring $\Lambda_{p}$ which, as $\pi_{*}$-module, is flat. The main objects of this section are (i) to compute the finitistic global dimension of $\Lambda_{p}$; and (ii) to realize $\Lambda_{p}$ as a stable homotopy module of some spectrum $L_{p}$.

\section{The finitistic grobal dimension of $\Lambda_{p}$.}

Before we state the main theorem, recall the theorem of KervaireMilnor [11].

THEOREM 3.1. For each prime $p$ and positive integer $j$, there exists $\alpha \in \Lambda_{p}$ such that $p^{j} \cdot \alpha=0$ and $p^{j-1} \cdot \alpha \neq 0$.

Let the notation be as in Definition 2.3. Then our main theorem in this section is the following

THEOREM 3.2. (i ) f.P.D $\left(\Lambda_{p}\right)=f \measuredangle \cdot P . D\left(\Lambda_{p}=0<F . P . D\left(\Lambda_{p}\right) \neq 0\right.$ (ii) $f \cdot W \cdot D\left(\Lambda_{p}\right)=f \ell \cdot W \cdot D\left(\Lambda_{p}\right)=0 \leqq F \cdot W \cdot D\left(\Lambda_{p}\right)$.

This theorem shows that the conjecture stated in $[9$, pp. $62,(2)]$ should exclude the case $f . P . D=0$.

The proof of this theorem follows that of Lemma 3.3 and 3.4 below.

LEMMA 3.3. $f \cdot P \cdot D\left(\Lambda_{p}\right)=f \ell \cdot P \cdot D\left(\Lambda_{p}\right)=0$.

Proof. By definition, it suffices to prove $f \iota \cdot P \cdot D\left(\Lambda_{p}\right)=0$. In order to prove this, let us assume, to the contrary, that $f \iota . P . D\left(\Lambda_{p}\right)>0$. Then from Corollary 2.5 there is a locally finitely generated $\Lambda_{p}$-module $A$ of projective dimension one. Moreover since $\Lambda_{p}$ is a (locally Noetherian) local ring, by Theorem 2.15. (ii), we have a free resolution

$$
0 \longleftarrow A \stackrel{\varepsilon}{\longleftarrow} F \longleftarrow K \longleftarrow 0
$$


with $K$ and $F$ being locally finitely generated. Also we can choose $K$ and $F$ so that $K \subset M \cdot F$, where $M$ is the Jacobson radical (= the unique maximal ideal) of $\Lambda_{p}$. For, let $\left\{a_{i}\right\}$ be a minimal generating system of $A$, let $F$ be a free module with generators $\left\{x_{i}\right\}$ (where $\operatorname{deg} x_{i}=\operatorname{deg} a_{i}$ ), and let $\varepsilon$ be a map sending $x_{i}$ to $a_{i}$. Now $\sum \alpha_{i} x_{i}$ is in $K$ if and only if $\sum \alpha_{i} \alpha_{i}=0$. Suppose $\alpha_{1}$ is not in $M$. Then $\alpha_{1}$ is a unit. Thus $a_{1}=-\alpha_{1}^{-1}\left(\sum_{i>1} \alpha_{i} e_{i}\right)$, which contradicts the minimality of $\left\{a_{i}\right\}$. Hence $\alpha_{1}$ and similarly $\alpha_{i}$ must be in $M$. Therefore $K$ is containd in $M \cdot F$ which is a direct sum $\bigoplus_{i=1}^{n}\left\{M_{i} \mid M_{i}=M\right\}$. Since $K$ is free, then $K \subset M \cdot F$ implies that $L_{\ell} \Lambda_{p} \subset \bigoplus_{i=1}^{n}\left\{M_{i} \mid M_{i}=M\right\}$, where $L_{\ell} \Lambda_{p}$ is the ring $\Lambda_{p}$ with degree lifted by $\ell$ (see Definition 2.17). Then we have

$$
L_{\ell} 1=\left(m_{1}, m_{2}, \cdots, m_{n}\right), m_{i} \in M .
$$

This implies that the annihilator of $m_{i}(i=1,2, \cdots, n)$ is also an annihilator of $L_{\ell} 1$, and therefore is zero. We will now show that the annihilator of $\left\{m_{i}\right\}$ is not zero.

Let $m_{i_{1}}, \cdots, m_{i_{s}}$ be those $m_{i}$ with degree $\geqq 1$. Let $p^{j}$ be the maximum additive order of $m_{i_{1}}, \cdots, m_{i_{s}}$. From Theorem 3.1, there is $\beta \in \Lambda_{p}$ such that $p^{j} \cdot \beta \neq 0$ and $p^{j+1} \cdot \beta=0$. Write $p^{j} \cdot \beta=\beta_{0}$. Then obviously $\beta_{0} \cdot m_{i_{k}}=\beta \cdot p^{j} \cdot m_{i_{k}}=0$, for $k=1, \cdots, s$, and $p \cdot \beta_{0}=0$. Note that the elements of degree 0 in the Jacobson radical $M$ of $\Lambda_{p}$ are of the form $t p / q$, where $p$ and $q$ are relatively prime. Thus $\beta_{0} \cdot m_{j}=$ $\beta_{0} \cdot t p / q=t / q \cdot p \cdot \beta_{0}=0$ for $j \neq i_{1}, \cdots, i_{s}$. This shows that $\beta_{0} \neq 0$ is a nonzero annihilator of $m_{i}, i=1, \cdots, n$. Hence the annihilator of $L_{\ell} 1$ is nonzero; contradiction. This concludes the proof of the lemma.

To prove the strict inequality of the theorem, we prove

LemMA 3.4. Let $R=\left\{R_{n}, n \geqq 0\right\}$ be a nonnegatively graded commutative ring. Then F.P.D $(R)=0$ implies that $R_{0}$, the 0th component of $R$, is an ungraded perfect ring.

Proof. From the proof of [3, Lemma 1.3], we see that $F \cdot P \cdot D(R)=$ 0 implies that every decreasing sequence of principal ideals $\alpha_{1} R \varsubsetneqq$ $\alpha_{1} \alpha_{2} R \varsubsetneqq \cdots \varsubsetneqq \alpha_{1} \cdots \alpha_{n} R \varsubsetneqq \cdots$ is finite, (deg $\left.\alpha_{i}=0\right)$. But observe that since we have chosen $\operatorname{deg} \alpha_{i}=0$, every principal ideal $\alpha_{1} \cdots \alpha_{n} R$ of $R$ gives rise to a principal $\alpha_{1} \cdots \alpha_{n} R_{0}$ of $R_{0}$, and vice versa. Thus the finiteness of the decreasing sequence $\left\{\alpha_{1} \cdots \alpha_{n} R\right\}$ implies that of $\left\{\alpha_{1} \cdots\right.$ $\left.\alpha_{n} R_{0}\right\}$. Then, by [3, Theorem P], $R_{0}$ is an ungraded perfect ring.

Proof of Theorem 3.2. The 0th component of $\Lambda_{p}$ is just the local ring $Z(p)$ obtained by localizing the $\operatorname{ring} Z$ of the integers at the 
prime ideal $p Z$, and $Z(p)$ is obviously not a perfect ring. Thus we conclude from Lemma 3.4 that F.P. $D\left(\Lambda_{p}\right) \neq 0$. Since $\Lambda_{p}$ is locally Noetherian, by Proposition 2.9. (ii),

$$
f \iota \cdot W \cdot D\left(\Lambda_{p}\right)=f \cdot W \cdot D\left(\Lambda_{p}\right)=f \iota \cdot P \cdot D\left(\Lambda_{p}\right)=0,
$$

while from its definition $F \cdot W \cdot D\left(\Lambda_{p}\right) \geqq 0$. Thus we prove the theorem.

II. Geometric realization.

The purpose of this section is to prove the following theorem.

THEOREM 3.5. There exists a spectrum $L_{p}$ such that $\pi_{*}\left(L_{p}\right)=\Lambda_{p}$ as $a \pi_{*}$-module.

The proof of this theorem is broken into lemmas.

Let $q_{1}, q_{2}, \cdots, q_{i}, \cdots$ be all the prime integers except $p$. Let $Q_{i}$ be the multiplicative system $Q_{i}=\left\{1, q_{i}, q_{i}^{2}, \cdots, q_{i}^{n}, \cdots\right\}$, and $\left(Q_{1}, \cdots, Q_{n}\right)$ be a multiplicative system generated by $Q_{i}, i=1,2, \cdots, n$. Since we have identified the ring $Z$ of integers with $\pi_{0}$, each $Q_{i}$ and hence $\left(Q_{1}, \cdots, Q_{n}\right)$ are multiplicative systems (of degree 0 ) in $\pi_{*}$. Hence, by $\S 1$. III, we have a sequence of local rings i.e.,

$$
\begin{aligned}
& \pi_{*}\left(Q_{1}\right) \text { is } \pi_{*} \text { localized at } Q_{1} \\
& \pi_{*}\left(Q_{1}, Q_{2}\right) \text { is } \pi_{*} \text { localized at }\left(Q_{1}, Q_{2}\right) \\
& \vdots \\
& \pi_{*}\left(Q_{1}, Q_{2}, \cdots, Q_{n}\right) \text { is } \pi_{*} \text { localized at }\left(Q_{1}, Q_{2}, \cdots, Q_{n}\right) .
\end{aligned}
$$

Note that the ring $Z=\pi_{0}$ is still naturally embedded in each $\pi_{*}\left(Q_{1}, Q_{2}, \cdots, Q_{n}\right)$. Thus it is legitimate to regard, say $Q_{n+1}$, as a multiplicative system (of degree 0 ) in $\pi_{*}\left(Q_{1}, Q_{2}, \cdots, Q_{n}\right)$. Hence we have the following lemma:

LEMMA 3.6. $\pi_{*}\left(Q_{1}, Q_{2}, \cdots, Q_{n+1}\right)$ is the local ring obtained by localizing $\pi_{*}\left(Q_{1}, \cdots, Q_{n}\right)$ at $Q_{n+1}$, and hence

$$
\pi_{*}\left(Q_{1}, \cdots, Q_{n+1}\right)=\lim _{\rightarrow} \pi_{*}\left(Q_{1}, \cdots, Q_{n}\right)
$$

where the direct limit "lim" is taken over the direct system:

$$
\pi_{*}\left(Q_{1}, \cdots, Q_{n}\right) \stackrel{q_{n+1}}{\longrightarrow} \pi_{*}\left(Q_{1}, \cdots, Q_{n}\right) \stackrel{q_{n+1}}{\longrightarrow} \cdots,
$$

in which $q_{n+1}$ is multiplication by the prime $q_{n+1}$.

Note also that there are natural "embedding" arising from 
localizations (see $\S 1$. III)

$$
f_{n}: \pi_{*}\left(Q_{1}, Q_{2}, \cdots, Q_{n}\right) \longrightarrow \pi_{*}\left(Q_{1}, Q_{2}, \cdots, Q_{n+1}\right), n \geqq 0
$$

where, $n=0, \pi_{*}\left(Q_{1}, Q_{2}, \cdots, Q_{n}\right)$ is interpreted as $\pi_{*}$. (The $f_{n}$ are not necessarily monomorphisms; see $\S 1$. III).

From the construction of localization it is not difficult to see that we have the following lemma.

Lemma 3.7. The sequence $\left\{\pi_{*}\left(Q_{1}, \cdots, Q_{n}\right), f_{n} \mid n=0,1,2, \cdots\right\}$ is a direct system of $\pi_{*}$-modules, with direct limit

$$
\Lambda_{p}=\lim \pi_{*}\left(Q_{1}, \cdots, Q_{n}\right) \text {. }
$$

Next we will construct geometric objects realizing these $\pi_{*}$ modules, namely,

LEMma 3.8. There exist a sequence of spectra $\left\{\mathscr{L}_{n}\right\}$ and a sequence of maps $\left\{g_{n}: \mathscr{L}_{n} \rightarrow \mathscr{L}_{n+1}\right\}$ such that, for $n \geqq 0$

$$
\pi_{*}\left(\mathscr{L}_{n}\right)=\pi_{*}\left(Q_{1}, Q_{2}, \cdots, Q_{n}\right) ;
$$

and

$$
\left(g_{n}\right)_{*}=f_{n}: \pi_{*}\left(Q_{1}, Q_{2}, \cdots, Q_{n}\right) \longrightarrow \pi_{*}\left(Q_{1}, \cdots Q_{n+1}\right)
$$

where $f_{n}$ is the map in Lemma 3.7.

Proof. Take $\mathscr{L}^{0}=S^{0}$ be the 0 -sphere spectrum. Assume inductively that the spectrum $\mathscr{L}_{n}$ is constructed such that

$$
\pi_{*}\left(\mathscr{L}_{n}\right)=\pi_{*}\left(Q_{1}, \cdots, Q_{n}\right) .
$$

Then we are going to construct $\mathscr{L}_{n+1}$ such that

$$
\pi_{*}\left(\mathscr{L}_{n+1}\right)=\pi_{*}\left(Q_{1}, \cdots, Q_{n+1}\right) .
$$

Namely, consider the following directed system

$$
\mathscr{L}_{n} \stackrel{q_{n}^{\prime}}{\longrightarrow} \mathscr{L}_{n} \stackrel{q_{n}^{\prime}}{\longrightarrow} \cdots \stackrel{q_{n}^{\prime}}{\longrightarrow} \mathscr{L}_{n} \longrightarrow \cdots
$$

where $q_{n}^{\prime}$ is the identity map multiplied by the prime $q_{n}$ (which is the $n$th prime listed in the beginning of this section $\S 3$. II). Then, by [6, pp. 22, 4.5], the directed system (3) gives rise to a spectrum $\mathscr{L}_{n+1}$ and a filtration $\left\{Y_{i}\right\}$ of $\mathscr{L}_{n+1}$ such that, for each $i$, there is a homotopy equivalence

$$
Y_{i} \simeq \mathscr{L}_{n}
$$


and such that there exists a homotopy commutative diagram

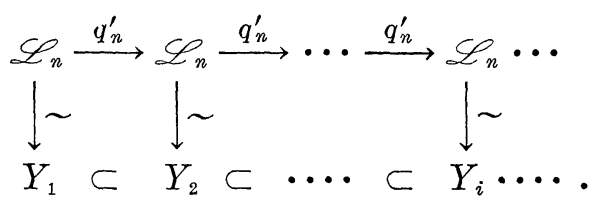

Thus, since the direct limit behaves well with respect to the stable homotopy group [6, pp. 21, 4.5], we have

$$
\pi_{*}\left(\mathscr{L}_{n+1}\right)=\pi_{*}\left(\lim _{\rightarrow} Y_{i}\right)=\lim _{\rightarrow} \pi_{*}\left(Y_{i}\right)=\lim _{\rightarrow} \pi_{*}\left(\mathscr{L}_{n}\right)
$$

where the first direct limit "lim" is taken over the bottom line of the above diagram (4), and the last "lim" is taken over (3). Note that we are assuming inductively that $\pi_{*}\left(\mathscr{L}_{n}\right)=\pi_{*}\left(Q_{1}, \cdots, Q_{n}\right)$. Hence (5) and Lemma 3.6, (1) imply that

$$
\pi_{*}\left(\mathscr{L}_{n+1}\right)=\pi_{*}\left(Q_{1} \cdots, Q_{n+1}\right) .
$$

Thus we have constructed $\mathscr{L}_{n+1}$.

Note that there is a natural projection

$$
g_{n}: \mathscr{L}_{n}=Y_{1} \longrightarrow \mathscr{L}_{n+1}=\lim _{\longrightarrow} Y_{i}
$$

which obviously induces a map

$$
\pi_{*}\left(\mathscr{L}_{n}\right)=\pi_{*}\left(Y_{1}\right) \rightarrow \pi_{*} \mathscr{L}_{n+1}=\lim _{\rightarrow} \pi_{*}\left(\mathscr{L}_{n}\right)
$$

that is just the map $f_{n}$. Thus we complete the proof of this lemma.

3.9. The proof of the Theorem 3.5.

Consider the directed system

$$
\mathscr{L}_{1} \stackrel{g_{0}}{\longrightarrow} \mathscr{L}_{1} \stackrel{g_{1}}{\longrightarrow} \cdots \longrightarrow \mathscr{L}_{n} \stackrel{g_{n}}{\longrightarrow} \cdots
$$

which is constructed in Lemma 3.8. Then again by [6, pp. 22, 4.5], this directed system gives rises to a spectrum $L_{p}$ such that

$$
\pi_{*}\left(L_{p}\right)=\lim _{\rightarrow} \pi_{*}\left(\mathscr{L}_{n}\right)
$$

where $\underset{\rightarrow}{\lim }$ is taken over (6). Then, by Lemma 3.8,

$$
\pi_{*}\left(L_{p}\right)=\lim _{\rightarrow} \pi_{*}\left(Q_{1}, \cdots, Q_{n}\right),
$$

and by Lemma 3.7

$$
\pi_{*}\left(L_{p}\right)=\Lambda_{p}
$$


Thus we have constructed a spectrum $L_{p}$ such that $\pi_{*}\left(L_{p}\right)=\Lambda_{p}$, and this completes the proof.

4. Stable homotopy ring. This section is the main body of the whole paper. The main theorems will be proved in first part, modulo a key computation. The key computation is in Theorem 2; we will prove this theorem in the second part.

\section{First let us prove}

THEOREM 4.1. Let $A$ be a locally finitely generated $\pi_{*}$-module having finite projective, as well as weak, dimension. Then $A$ is a free $\pi_{*}$-module and hence is realizable as a stable homotopy module $\pi_{*}(Y)$ of a wedge $Y$ of spheres.

REmark. The condition that $A$ is locally finitely generated is necessary, see Theorem 4.4 below.

Proof. This theorem essentially depends on the next theorem, so we will give a proof modulo Theorem 2:

Let $A$ be a locally finitely generated $\pi_{*}$-module. Then we will show that $P . d_{\pi_{*}} A<\infty$ implies that $A$ is a free $\pi_{*}$-module. From Corollary 2.5, we see that it is sufficient to prove this for the case $P . d_{\pi *} A \leqq 1$. Let

$$
0 \longleftarrow A \longleftarrow F_{0} \stackrel{d_{1}}{\longleftarrow} F_{1} \longleftarrow 0
$$

be a locally finitely generated free resolution (by Theorem 2.15 (ii)). Then, by Theorem 2.18, we can realize $F_{0}, F_{1}$ and $d_{1}$ by wedges of spheres $N_{0}, N_{1}$ and a map $f: N_{0} \rightarrow N_{1}$; that is, $\pi_{*}\left(N_{0}\right) \cong F_{0}, \pi_{*}\left(N_{1}\right) \simeq F_{1}$ and $d_{1}=f_{*}$. Let $C_{f}$ be the mapping cone of $f$, then $C_{f}$ has a finite skeleton for each dimension (since $N_{0}$ and $N_{1}$ were chosen to have only fiinitely many spheres at each dimension). Since the map $d_{1}=f_{*}$ is a monomorphism, the Puppe sequence (of the mapping cone sequence $N_{0} \rightarrow N_{1} \rightarrow C_{f}$ ) reduces to a short exact sequence

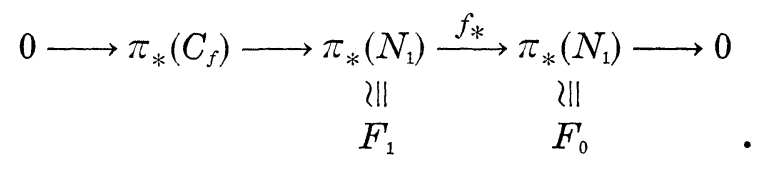

Comparing this with the other sequence above (i.e. the free resolution of $A$ ), we conclude that $\pi_{*}\left(C_{f}\right) \cong A$; hence $P . d_{\pi_{*}}\left(\pi_{*}\left(C_{f}\right)\right)=P . d_{\pi_{*}} A \leqq 1$. On the other hand, from the next theorem (See Theorem 2 below), 
$P . d_{\pi_{*}} A=P . d_{\pi_{*}}\left(\pi_{*}\left(C_{f}\right)\right)=0$ or $\infty$. Therefore we have $\left.P . d_{\pi_{*}}\left(\pi_{*} C_{f}\right)\right)=$ $P . d_{\pi_{*}} A=0$; hence, by Corollory 2.14 (1), $A$ is free; and by Theorem 2.18 it is realizable by a wedge of spheres. This proves the theorem. The proof of the next theorem is very long so we will only state the theorem here; the proof will be given later.

THEOREM 4.2. Let $Y$ be a connected spectrum having a finite skeleton at each dimension. Then the projective, as well as weak, dimension of $\pi_{*}(Y)$ as $\pi_{*}$-module is infinite unless $Y$ is a wedge of spheres.

THEOREM 4.3. (i) f.P.D $\left(\pi_{*}\right)=f \ell \cdot P \cdot D\left(\pi_{*}\right)=0<F \cdot P \cdot D\left(\pi_{*}\right) \neq 0$

(ii) $f \cdot W \cdot D\left(\pi_{*}\right)=f \ell \cdot W \cdot D\left(\pi_{*}\right)=0 \leqq F \cdot W \cdot D\left(\pi_{*}\right)$.

Proof. f.P. $D\left(\pi_{*}\right)=f \iota \cdot P \cdot D\left(\pi_{*}\right)=f \cdot W \cdot D\left(\pi_{*}\right)=f \iota \cdot W \cdot D\left(\pi_{*}\right)=0$ follows from Theorem 4.1. $F \cdot W \cdot D\left(\pi_{*}\right) \geqq 0$ follows from the definition. F.P.D $\left(\pi_{*}\right)>0$ follows from Lemma 3.4.

THEOREM 4.4. There is a stable homotopy module $\pi_{*}(X)$ such that $P . d_{\pi_{*}}\left(\pi_{*}(X)\right)=1$.

Proof. By Theorem 4.3, F.P.D $\left(\pi_{*}\right)>0$. It then follows from Corollary 2.5 that there is a $\pi_{*}$-module, say $A$, such that $P . D_{\pi_{*}}(A)=1$. Let

$$
0 \longleftarrow A \longleftarrow F_{0} \longleftarrow F_{1} \longleftarrow 0
$$

be a free resolution of $A$, which exists, by Theorem 2.15, (ii). Then, by Theorem 2.18, we can realize $F_{0}, F_{1}$ and $d_{1}$ by wedges of spheres $N_{0}, N_{1}$ and a map $f: N_{1} \rightarrow N_{0}$, i.e., $\pi_{*}\left(N_{0}\right) \cong F_{0} \pi_{*}\left(N_{1}\right) \cong F_{1}$ and $f_{*}=d_{1}$. Let $L_{1} \stackrel{f}{\rightarrow} N_{0} \rightarrow C_{f}$ be the mapping cone sequence of $f$, then the Puppe sequence reduces to a short exact sequence

$$
0 \longleftarrow \pi_{*}\left(C_{f}\right) \longleftarrow \pi_{*}\left(N_{0}\right) \stackrel{f_{*}}{\longleftarrow} \pi_{*}\left(N_{1}\right) \longleftarrow 0,
$$

since $f_{*}=d_{1}$ is a monomorphism. By comparing the two short exact sequences, we see that $\pi_{*}\left(C_{f}\right) \cong A$. Setting $X=C_{f}$ finishes the proof.

REMARK. From [14], we can show that the spectrum $L_{p}$, which is constructed in Theorem 3.5, is such a spectrum.

From Theorem 4.3 and [3, Corollary 5.6], we have

THeOREM 4.5. (1) Every finitely generated proper ideal of $\pi_{*}$ has nonzero annihilator 
(ii) A finitely generated projective submodule of a projective module is always a direct summand.

THEOREM 4.6. The projective as well as weak, dimension of an ideal in $\pi_{*}$ is infinite.

Proof. Let $I \subset \pi_{*}$ be a proper ideal. Let us assume that $W \cdot d_{\pi_{*}} I \leqq$ $P . d_{\pi_{*}} I<\infty$. Then, by Theorem 4.3, P. $D_{\pi_{*}} I=0$; and by Corollary 2.14, $I$ is free. Thus the annihilator of $I$ has to be zero and this contradicts Theorem 4.5. Therefore $P . d_{\pi_{*}} I=\infty$.

THEOREM 4.7. Let ${ }_{p} \pi_{*}$ be the subring of $\pi_{*}$ consisting of $\pi_{0}$ and p-primary component of $\pi_{n}, n \geqq 1$. Then the global dimension of ${ }_{p} \pi_{*}$ is infinite.

Proof. From the "slide product" (e.g. see [1] or [12]) representation of functor Tor, it is easy to see that the functors $\operatorname{Tor}^{\pi_{*}}\left(-, Z_{p}\right)$ and $\operatorname{Tor}^{p^{\pi *}}\left(-, Z_{p}\right)$ are equal. Since $Z_{p}$, the integers $\bmod p$, has weak dimension equal to $\infty$, so there are infinitely many $n$ such that, $\operatorname{Tor}_{n, *}^{p \pi *}\left(-, Z_{p}\right) \neq 0$. Hence $G \cdot D\left({ }_{p} \pi_{*}\right)=\infty$.

II. Next we will devote ourselves to the proof of Theorem 2 . First let us recall a Kunneth type theorem for stable homotopy groups as a generalized theory. The details are in [1] or [12]. Let $W \wedge Y$ be the smash product and let $\pi_{*}(X, W)$ be the graded abelian group of the stable homotopy classes of maps from $X$ to $W$. Let $h_{\alpha}: X^{\alpha} \rightarrow Y$ be a family of maps, and let $\vee_{\alpha} X^{\alpha}$ be the wedge of $X^{\alpha \prime} s$. Then there is a map

$$
h: X^{\alpha} \longrightarrow Y
$$

such that its restriction on $X^{\alpha}$ is $h_{\alpha}$, i.e., $h \mid X^{\alpha}=h_{\alpha}$. We will call this map $h$ the wedge of $h_{\alpha}$ and denote it by $h=\vee_{\alpha} h_{\alpha}$.

THEOREM 4.8. Let $X, Y, W$ be spectra. Then there exist spectral sequence $\left\{E_{*}^{\gamma}, *\right\}$ and $\left\{\tilde{E}_{*}^{\gamma}, *\right\}$ with

$$
\begin{aligned}
& E_{*}^{2},{ }^{*}=\operatorname{Tor}^{\pi *}\left(\pi_{*}(W), \pi_{*}(Y)\right) ; \\
& \widetilde{E}_{*}^{2},{ }_{*}=\operatorname{Tor}^{\pi *}\left(\pi_{*}(W), \pi^{*}(X)\right)
\end{aligned}
$$

and if $X$ is a finite spectrum and $Y, Z$ are connected spectra, then both spectral sequence converge

$$
\begin{aligned}
& E_{*}^{r}, \pi_{*}(W \wedge Y) ; \\
& \widetilde{E}_{*}^{\gamma}, \rightleftharpoons \pi_{*}(X, W) .
\end{aligned}
$$


Moreover the spectral sequences are functor of their arguments and the filtrations

$$
\begin{aligned}
& F^{o} \subset F^{1} \subset \cdots \subset F^{n} \cdots ; \cup F^{n}=\pi_{*}(W \wedge Y) ; \\
& \widetilde{F}^{o} \subset \widetilde{F}^{1} \subset \cdots \subset \widetilde{F}^{n} \cdots ; \cup \widetilde{F}^{n}=\pi_{*}(X, W),
\end{aligned}
$$

which satisfy

$$
\begin{aligned}
& F^{n} / F^{n-1}=E_{n, *}^{\infty} \\
& \widetilde{F}^{n} / \widetilde{F}^{n-1}=\widetilde{E}_{n, *}^{\infty},
\end{aligned}
$$

are given by

$$
\begin{aligned}
& F^{n}=\operatorname{Ker}\left\{\pi_{*}(W \wedge Y) \longrightarrow \pi_{*}\left(W_{n} \wedge Y\right)\right\} \\
& \widetilde{F}^{n}=\operatorname{Ker}\left\{\pi_{*}(X, W) \longrightarrow \pi_{*}\left(X, W_{n}\right)\right\}
\end{aligned}
$$

where $W_{n}$ is defined inductively as follows: Let $W_{-1}=W$, and assume that $W_{n}$ is defined. Let $\left\{g_{\alpha}: S^{n_{\alpha}} \rightarrow W_{n}\right\}$ be the set of generators of $\pi_{*}\left(W_{n}\right)$ as a $\pi_{*}$-module. Let $M_{n+1}=\vee_{\alpha} S^{n_{\alpha}}$, and let $f_{n+1}=\vee_{\alpha} g_{\alpha}: M_{n+1} \rightarrow$ $W_{n}$ be the wedge of maps $g_{\alpha}$. Then $W_{n+1}$ is defined to be the mapping cone of $f_{n+1}$.

REMARK. Let $\pi_{*}(X) \otimes \pi_{*}(Y)$ be the tensor product over $\pi_{*} \cdot{ }^{1}$ Then the edge homomorphism of the spectral sequence $\left\{E_{*, *}^{r}\right\}$ in the above theorem is a map

$$
\pi_{*}(X) \otimes \pi_{*}(Y) \longrightarrow \pi_{*}(X \wedge Y)
$$

which sends $f \otimes g$ into $f \wedge g$ for $f \in \pi_{*}(X)$ and $g \in \pi_{*}(Y)$. We will call this map smash product (This map is stronger than the usual "smash product", because we require the tensor product be over $\pi_{*}$ ).

CoROLlary 4.9. If $\pi_{*}(W)$ is a flat $\pi_{*}$-module, then the smash product

$$
\pi_{*}(W) \otimes \pi_{*}(Y) \longrightarrow \pi_{*}(W \wedge Y)
$$

is an isomorphism.

CoRollary 4.10. Let $\pi_{*}(W)$ be a flat $\pi_{*}$-module. Then all the primary and higher order homology operations of degree $\geqq 1$ are all zero on $H_{*}(W)$.

Proof. The notations are the same as Theorem 4.8. Since $\pi_{*}(W)$ is flat, the spectral sequence $\left\{E_{*, *}^{r}\right\}$ in the Theorem 4.8 has

\footnotetext{
1 Throughout the whole paper $\otimes$ will always mean tensor product over the ring $\pi_{*}$.
} 


$$
E_{n, *}^{2}=\operatorname{Tor}_{n, *}^{\pi *}\left(\pi_{*}(W), \pi_{*}(Y)\right)=0 \quad n \neq 0
$$

Hence

$$
E_{n, *}^{\infty}=0, \text { for } n \neq 0
$$

In other words

$$
F^{0}=F^{1}=F^{2}=\cdots=\bigcup_{i} F^{i}=\pi_{*}(W \wedge Y)
$$

or equivalently,

$$
F^{0}=\pi_{*}(W \wedge Y)=\operatorname{Ker}\left\{\pi_{*}(W \wedge Y) \longrightarrow \pi_{*}\left(W_{0} \wedge Y\right)\right\}
$$

That is,

$$
\pi_{*}(W \wedge Y) \longrightarrow \pi_{*}\left(W_{0} \wedge Y\right) \text { is a zero map . }
$$

Then by the Puppe sequence of the mapping cone sequence $M_{0} \rightarrow$ $W \rightarrow W_{0}$ (constructed in the statement of Theorem 4.8), we have an epimorphism

$$
\pi_{*}\left(M_{0} \wedge Y\right) \longrightarrow \pi_{*}(W \wedge Y) \longrightarrow 0
$$

Take $Y$ to be the Eilenberg-MacLane spectrum $K(G)$, then the above epimorphism reduces to the epimorphism

$$
H_{*}\left(M_{0} ; G\right) \longrightarrow H_{*}(W ; G) \longrightarrow 0
$$

where $G$ is any coefficient group. Since $M_{0}$ is a wedge of spheres (see Theorem 4.8), all the homology operations of degree $\geqq 1$ are zero on $H_{*}\left(M_{0}\right)$. Thus, by naturality of homology operations, all the homology operations of degree $\geqq 1$ on $H_{*}(W)$ are zero.

Next we will look at the Spaniar-Whithead duality and its relation with cohomology (homology) operations. Let $X$ be a finite spectrum (roughly speaking, that is a spectrum with finite number of stable cells; see [6, pp. 7, C. 7] for precise definition). Then, from [6, pp. 33, K. 12] we can choose its Spaniar-Whithead dual $D X$ to be finite too. Then it is obvious to observe that.

Lemma 4.11. Let $X$ be a finite spectrum. Then $X$ is a wedge of spheres if and only if $D X$ is.

Let us recall from [19] the cohomology operations. Let $X$ be an arbitrary spectrum, and let $\alpha$ be one of the lowest order nonzero cohomology operations on $H^{*}(X)$. Then $\alpha$ can be represented as a differential of the spectral sequence obtained from some Postnikov system. More precisely, let 


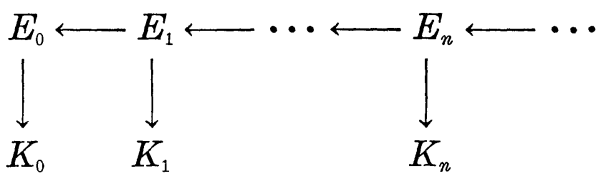

be a Postnikov system, where $K_{i}$ are Eilenberg-MacLane objects. By applying the functor $\pi_{*}(X,-)$ to the system we get a spectral sequence witn $E_{*, *}^{1}=\pi_{*}\left(X, K_{*}\right)=H^{*}\left(X ; K_{*}\right)$. Then since $\alpha$ is the lowest order nonzero cohomology operation (and hence has zero indeterminacy), the differential $\alpha$ representing this operation is a non-zero group-homomorphism

$$
\alpha: H^{q}\left(X ; K_{n_{1}}\right) \longrightarrow H^{q+r}\left(X ; K_{n_{2}}\right) .
$$

Next observe that $H^{*}\left(X, K_{n}\right)=\pi_{*}\left(D X \wedge K_{n}\right)=H_{*}\left(D X ; K_{n}\right)$, by SpaniarWhithead duality (see [19]). Then the spectral sequence can be written as

$$
E_{*, *}^{1}=H^{*}\left(X ; K_{*}\right)=H_{*}\left(D X ; K_{*}\right)
$$

and hence $\alpha$ is a nonzero homology operation on $H_{*}(D X)$. Thus we have proved the following proposition.

Proposition 4.12. Let $X$ be a finite spectrum. Then there is a nonzero cohomology operation of degree $\geqq 1$ on $H^{*}(X)$ if and only if there is a nonzero homology operation of degree $\geqq 1$ on $H_{*}(D X)$.

We need an algebraic Lemma on localization.

LEMMA 4.13. Let $\delta: G_{1} \rightarrow G_{2}$ be a nonzero homomorphism of abelian groups from $G_{1}$ to $G_{2}$. Then there is a prime integer $p$ such that

$$
\delta \otimes_{Z} Z(p): G_{1} \otimes_{z} Z(p) \rightarrow G_{2} \otimes_{z} Z(p)
$$

is a nonzero homomorphism, where $Z(p)$ is the local ring obtained by localizing the ring $Z$ of integers at the prime ideal $p Z$, and $\otimes_{z}$ is the tensor product of abelian groups.

Proof. It is sufficient to show that there is a prime $p$ such that $(\operatorname{Im} \delta) \otimes_{Z} Z(p) \neq 0$. Let $C \subset \operatorname{Im} \delta$ be a cyclic subgroup of $\operatorname{Im} \delta$, then $C \otimes_{Z} Z(p) \subset \operatorname{Im} \delta \otimes_{z} Z(p)$. (Note that $Z(p)$ is a torsion free group; hence the inclusion is preserved). Thus it is sufficient to show that for any cyclic group $C$, there is some $Z(p)$ such that $C \otimes_{Z} Z(p) \neq 0$; and this is obvious.

PRoposition 4.14. Let all the homology operations of degree $\geqq 1$ be zero on $H_{*}(Y)$. Then the integral homology group $H_{*}(Y ; Z)$ is a 
torsion free abelian group.

Proof. Suppose $H_{m}=H_{m}(Y ; Z)$ be the lowest dimension group which has torsion. Let $G$ be a group such that $\operatorname{Tor}^{z}\left(H_{m}, G\right) \neq 0$. Let<smiles>O[Te][Te][Te][Te]O</smiles>

be a $Z$-free resolution of $G$. Then we have the exact sequence of Tor:

$$
\begin{aligned}
0=\operatorname{Tor}^{Z}\left(H_{m}, P_{0}\right) & \longrightarrow \operatorname{Tor}^{Z}\left(H_{m}, G\right) \longrightarrow H_{m} \otimes_{Z} P_{1} \stackrel{H_{m} \otimes_{z} d_{1}}{\longrightarrow} H_{m} \otimes P_{0} \\
& \longrightarrow H_{m} \otimes G_{Z} \longrightarrow 0
\end{aligned}
$$

Since $\operatorname{Tor}^{Z}\left(H_{m}, G\right) \neq 0$, so $H_{m} \otimes_{z} d_{1}$ can not be monomorphic. On the other hand, the long exact sequence of homology groups associated to $(1)$ is

$$
\cdots \longrightarrow H_{m+1}(Y, G) \stackrel{\beta}{\longrightarrow} H_{m}\left(Y, P_{1}\right) \stackrel{d_{1}^{\prime}}{\longrightarrow} H_{m}\left(Y, P_{2}\right) \longrightarrow \cdots .
$$

Since $P_{i}$ are free, so $H_{m}\left(Y, P_{i}\right)=H_{m} \otimes_{Z} P_{i}$ and $d_{1}^{\prime}=H_{m} \otimes_{z} d_{1}$. Therefore $d_{1}^{\prime}=H_{m} \otimes_{z} d_{1}$ is not a monomorphism. Thus, by exactness, the Bockstein operator $\beta$ is not zero. This proves the Proposition.

Proposition 4.15. Let $L_{p}$ be the spectrum constructed in Theorem 3.5. Then all the primary and higher order homology operations of degree $\geqq 1$ are zero on $H_{*}(Y)$ if all the primary and higher order homology operations of degree $\geqq 1$ are zero on $H_{*}\left(Y \wedge L_{p}\right)$ for every prime p.

Proof. Let us assume, to the contrary, that there are nonzero homology operations of degree $\geqq 1$ on $H_{*}(Y)$. Let $\alpha$ be one of the lowest order of nonzero homology operations of degree $\geqq 1$. Then $\alpha$ can be expressed as a differential of the spectral sequence of the following exact couple

$$
\begin{gathered}
\sum_{n} \pi_{*}\left(Y \wedge E_{n}\right) \longrightarrow \sum_{n} \pi_{*}\left(Y \wedge E_{n-1}\right) \\
\sum_{n} \pi_{*}\left(Y \wedge \stackrel{\swarrow}{K_{n}}\right)=\sum_{n} H_{*}\left(Y ; K_{n}\right)
\end{gathered}
$$

where $\left\{E_{n}\right\}$ is a Postnikov system and for each $n, K_{n}$ is an EilenbergMacLane spectrum, Since $\alpha$ is the lowest order nonzero operation (and hence the indeterminacy is zero), so the differential, which represents this operation $\alpha$, gives rise to a nonzero group homomorphism 
$\alpha^{\prime}$ on $E_{q_{1}, n_{1}}^{1}-$ component, i.e.,

$$
\alpha^{\prime}: H_{q_{1}}\left(Y ; K_{n_{1}}\right)=E_{q_{1}, n_{1}}^{1} \longrightarrow H_{q_{2}}\left(Y ; K_{n_{2}}\right)=E_{q_{2}, n_{2}}^{1}
$$

is a nonzero homomorphism of groups. Observe that $\pi_{*}\left(L_{p}\right)=\Lambda_{p}$ is a flat $\pi_{*}$-module, therefore

$$
\begin{aligned}
\sum \pi_{*}\left(Y \wedge E_{n}\right) \otimes \Lambda_{p} \longrightarrow \sum \pi_{*}\left(Y \wedge E_{n}\right) \otimes \Lambda_{p} \\
\sum \pi_{*}\left(Y \wedge K_{n}\right) \otimes \Lambda_{p}=\sum H_{*}\left(Y ; K_{n}\right) \otimes \Lambda_{p}
\end{aligned}
$$

is still an exact couple.

Moreover, from properties of tensor product, it is readily seen that

$$
H_{*}\left(Y ; K_{n}\right) \otimes \Lambda_{p}=H_{*}\left(Y ; K_{n}\right) \otimes_{z} Z(p), \text { as groups , }
$$

where $Z(p)$ is the ring $Z$ localized at $p Z$. Thus the differential $\alpha \otimes \Lambda_{p}$ of (3), when it is restricted to $E_{q_{1}, n_{1}}^{1}$, is just a homomorphism of groups

$$
\alpha^{\prime} \otimes_{z} Z(p): H_{q_{1}}\left(Y ; K_{n_{1}}\right) \otimes_{z} Z(p) \longrightarrow H_{q_{2}}\left(Y ; K_{n_{2}}\right) \otimes_{Z} Z(p) .
$$

Then, by Lemma 4.13 , we can choose a prime $p$ such that

$$
\alpha^{\prime} \otimes_{z} Z(p) \neq 0 \text {. }
$$

In other words, there is some prime $p$ such that the differential

$$
\alpha \otimes \Lambda_{p} \neq 0
$$

is not a zero differential in (3), since $\alpha \otimes \Lambda_{p} \mid E_{q_{1}, n_{1}}^{1}=\alpha^{\prime} \otimes_{Z} Z(p)$.

Next, note that the exact couple (3), by Corollary 4.9 , can be written as

$$
\begin{aligned}
\sum \pi_{*}\left(Y \wedge \underset{E_{n}}{E_{n}} \wedge L_{p}\right) \longrightarrow \sum \pi_{*}\left(Y \wedge E_{n} \wedge L_{p}\right) \\
\sum \pi_{*}\left(Y \wedge K_{n} \wedge L_{p}\right)=\sum H_{*}\left(Y \wedge L_{p} ; K_{n}\right) .
\end{aligned}
$$

Therefore the differential $\tilde{\alpha}$ of (5), which corresponds to the differential $\alpha \otimes \Lambda_{p}$ of (3), is not a zero differential, i.e., by (4) we have,

$$
0 \neq \tilde{\alpha}=\alpha \otimes \Lambda_{p}: H_{q_{1}}\left(Y \wedge L_{p} ; K_{n_{1}}\right) \longrightarrow H_{q_{2}}\left(Y \wedge L_{p} ; K_{n_{2}}\right) \text {. }
$$

Moreover $\tilde{\alpha}$ can be regarded as a homology operation (of degree $\geqq 1$ ) on $H_{*}\left(Y \wedge L_{p}\right)$; thus $\tilde{\alpha} \neq 0$ contradicts the assumtion of the proposition. This completes the proof.

We also need a well known folk theorem about F. Peterson's work on detecting maps by cohomology operations (e.g. see [13]). 
THEOREM 4.16. A connected spectrum is a wedge of spheres if and only if all the primary and higher order cohomology operations of degree $\geqq 1$ are zero.

Now we are ready for the proof of Theorem 2 .

4.17. The proof of Theorem 2.

From Example 2.8 and Proposition 2.9, we see that it is sufficient to show only for the projective dimension case. Let us assume that $P . d_{\pi *}\left(\pi_{*}(Y)\right)<\infty$. Then from standard homological algebra (e.g. see [17]) we have

$$
P . d_{\Lambda_{p}}\left(\pi_{*}(Y) \otimes \Lambda_{p}\right)<\infty .
$$

By Theorem 3.2, fŁ.P. $D\left(\Lambda_{p}\right)=0$. It then follows that

$$
P . d_{\Lambda_{p}}\left(\pi_{*}(Y) \otimes \Lambda_{p}\right)=0 \text {. }
$$

Thus, by Corollary 2.14, $\pi_{*}(Y) \otimes \Lambda_{p}$ is a free $\Lambda_{p}$-module. Moreover, by Corollary 4.9, we have

$$
\pi_{*}\left(Y \wedge L_{p}\right)=\pi_{*}(Y) \otimes \Lambda_{p},
$$

where $L_{p}$ is the spectrum constructed in Theorem 3.5, and $\pi_{*}\left(L_{p}\right)=$ $\Lambda_{p}$. Thus $\pi_{*}\left(Y \wedge L_{p}\right)$, a free $\Lambda_{p}$-module, is a flat $\pi_{*}$-module. Then, by Corollary 4.10 , all the homology operations of degree $\geqq 1$ are all zero on $H_{*}\left(Y \wedge L_{p}\right)$; and this is true for every prime $p$. By Proposition 4.15 , all the homology operations of degree $\geqq 1$ are zero on $H_{*}(Y)$.

Since $Y$ is connected, there is a homology decomposition, or coPostnikov system $\left\{Y_{n}\right\}$ (e.g. see [8])

$$
\begin{aligned}
& Y_{m} \subset Y_{m+1} \subset \cdots \subset Y_{n} \subset \cdots ; \bigcup_{n \geqq m} Y_{n}=W \simeq Y \\
& \begin{array}{ccc}
\uparrow_{k_{m}^{\prime}} & \uparrow k_{m+1} & \uparrow k_{n}^{\prime} \\
N_{m} & N_{m+1} & N_{n}
\end{array}
\end{aligned}
$$

such that

(i) $H_{q}(Y ; Z) \cong H_{q}\left(Y_{n} ; Z\right) \quad q \leqq n$

(ii) $H_{q}\left(Y_{n} ; Y\right)=0 \quad q>n$

(iii) $N_{n}$ is the Moore space of type $\left(H_{n+1}(Y), n\right)$

(iv) $Y_{p+1}=Y_{n} \cup k_{n}^{\prime} C N_{n}$, the mapping cone of $k_{n}^{\prime}$.

(v) $k_{*}^{\prime}: H_{*}\left(N_{n}\right) \longrightarrow H_{*}\left(Y_{n}\right)$ is 0 .

Note that, in the last paragraph, we have shown that all the homology operations of degree $\geqq 1$ are zero on $H_{*}(Y)$. Thus, by Proposition 4.14, $H_{*}(Y ; Z)$ is torsion free. By the universal coefficient theorem, 


$$
H_{*}(Y ; G)=H_{*}(Y ; Z) \otimes_{Z} G
$$

Thus (i) and (ii) of (6) can be improved to

(i') $H_{q}(Y ; G) \cong H_{p}\left(Y_{n} ; G\right) \quad q \leqq n$

$$
\text { (ii') } H_{q}(Y ; G)=0 \quad q>n \text {. }
$$

Since all the homology operations (of degree $\geqq 1$ ) are all zero on $H_{*}(Y)$, by (i') and (ii'), we see immediately that all the homology operations of degree $\geqq 1$ are also zero on $H_{*}\left(Y_{n}\right)$ for every $n$. Thus, by Proposition 4.12, all the cohomology operations of degree $\geqq 1$ are all zero on $H^{*}\left(D Y_{n}\right)$ for every $n$, where $D Y$ is Spaniar-Whithead dual of $Y$. By Theorem 4.16, $D Y_{n}$ is a wedge spheres for each $n$. Recall that, by assumption, $Y$ has finite skeleton at each dimension. Therefore we can choose each $Y_{n}$ in (6) to be finite. Thus, by Lemma 4.11 and the fact $D D Y_{n} \simeq Y_{n}[6, \mathrm{pp} .33, \mathrm{~K}$. 12], we can conclude that each $Y_{n}$ is a wedge of sphere (since we have just shown above that $D Y_{n}$ is a wedge of spheres). Thus, by Peterson's theorem on detecting map, we conclude that $k_{n-1}^{\prime}$ is null-homotopic; and this is true for every $n$. Hence $Y$ is a wedge of spheres.

\section{REFERENCES}

1. J. F. Adams, Lectures on generalized cohomology, Lectures notes in Math. No, 99, Category theory, homology theory and their application III, pp. 1-138, Spring-Verlag 1968.

2. Adams, Stable homotopy theory, Lecture Notes in Math. No. 3 Spring-Verlag 1964.

3. H. Bass, Finitistic dimension and homological generalization of semi-primary ring, Trans. Amer. Math. Soc., 95 (1960), 466-488.

4. - Big projective modules are free. Illinois J. Math. (1963), 24-31.

5. —. Algebraic K-Theory, Benjamin 1968.

6. J. Boardman, Stable homotopy theory, Mimeograph notes, Univ. of Warick, 1965.

7. P. Freyd, Stable homotopy, Proc. of LaJolla Conf. pp. 121-172, Spring-Verlag 1965.

8. P. Hilton, Homotopy theory and duality, Gordon and Breach, 1965, New York 1964.

9. J. P. Jans, Rings and homology, Holt, Rinehart, and Winston, New York 1964.

10. D. W. Kahn, On stable homotopy modules I, Invent. Math. I, (1966), 375-379.

11. M. Kervaire and J. Milnor, Bernoulli numbers, homotopy groups and a theorem of Rohlin, Proc. Int. Congress, Edinborough, 1958.

12. T. Y. Lin, A spectral sequence in stable homotopy category, Thesis, Yale Univ. 1968.

13. - Cohomological characterization of stable spheres (unpublished).

14. Homological dimensions of $\pi_{*}$-modules and their geometric characterization (in preparation).

15. P. May, The Algebraic Eilenberg-Moore spectral Sequence (to appear).

16. - Homological algebra, lectures, Yale Univ. 1965.

17. D. G. Northcott. An introduction to homological algebra. Cambridge Univ. Press, New York. 1960.

18. O. Ore, Linear equation in non-commutative fields, Ann. of Math. 32 (1931), 463-477.

19. F. Peterson, Functional cohomology operations, Trans. Amer. Math. Soc., 86 (1957), 197-211.

20. Toda, Composition method in homotopy group of spheres, Ann. of Math, Studies, No. 49, Princeton, 1962. 
Received June 16, 1970 and in revised form November 9, 1970. The preparation of this paper was sponsored partly by the National Science Foundation Grant GP-21062. The abstract was announced in the Notices of AMS, February 1970, \#70-G37.

PURdue UNIVERSITY 



\section{PACIFIC JOURNAL OF MATHEMATICS}

\section{EDITORS}

H. SAMELSON

Stanford University

Stanford, California 94305

C. R. Hовву

University of Washington

Seattle, Washington 98105
J. DUGUNDJI

Department of Mathematics

University of Southern California

Los Angeles, California 90007

RICHARD ARENS

University of California

Los Angeles, California 90024

\section{ASSOCIATE EDITORS}
E. F. BECKENBACH
B. H. NeumanN
F. WOLF
K. YoSHIDA

\section{SUPPORTING INSTITUTIONS}

UNIVERSITY OF BRITISH COLUMBIA

CALIFORNIA INSTITUTE OF TECHNOLOGY

UNIVERSITY OF CALIFORNIA

MONTANA STATE UNIVERSITY

UNIVERSITY OF NEVADA

NEW MEXICO STATE UNIVERSITY

OREGON STATE UNIVERSITY

UNIVERSITY OF OREGON

OSAKA UNIVERSITY

UNIVERSITY OF SOUTHERN CALIFORNIA
STANFORD UNIVERSITY

UNIVERSITY OF TOKYO

UNIVERSITY OF UTAH

WASHINGTON STATE UNIVERSITY

UNIVERSITY OF WASHINGTON

AMERICAN MATHEMATICAL SOCIETY CHEVRON RESEARCH CORPORATION NAVAL WEAPONS CENTER 


\section{Pacific Journal of Mathematics}

\section{Vol. 38, No. $1 \quad$ March, 1971}

Bruce Alan Barnes, Banach algebras which are ideals in a Banach algebra ..... 1

David W. Boyd, Inequalities for positive integral operators............... 9

Lawrence Gerald Brown, Note on the open mapping theorem .............. 25

Stephen Daniel Comer, Representations by algebras of sections over Boolean

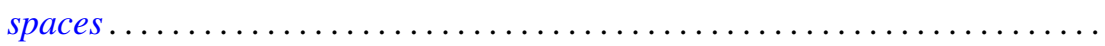

John R. Edwards and Stanley G. Wayment, On the nonequivalence of

conservative Hausdorff methods and Hausdorff moment sequences ........

P. D. T. A. Elliott, On the limiting distribution of additive functions $(\bmod 1) \ldots \ldots$

Mary Rodriguez Embry, Classifying special operators by means of subsets

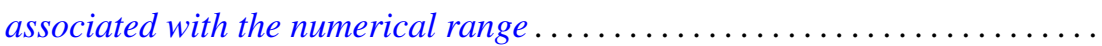

Darald Joe Hartfiel, Counterexamples to a conjecture of G. N. de Oliveira ......

C. Ward Henson, A family of countable homogeneous graphs...............

Satoru Igari and Shigehiko Kuratsubo, A sufficient condition for

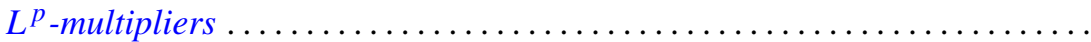

William A. Kirk, Fixed point theorems for nonlinear nonexpansive and

generalized contraction mappings............................

Erwin Kleinfeld, A generalization of commutative and associative rings ...... 95

D. B. Lahiri, Some restricted partition functions. Congruences modulo $11 \ldots \ldots 103$

T. Y. Lin, Homological algebra of stable homotopy ring $\pi *$ of spheres ....... 117

Morris Marden, A representation for the logarithmic derivative of a meromorphic function...........................

John Charles Nichols and James C. Smith, Examples concerning sum properties for metric-dependent dimension functions . .

Asit Baran Raha, On completely Hausdorff-completion of a completely

Hausdorff space.

M. Rajagopalan and Bertram Manuel Schreiber, Ergodic automorphisms and affine transformations of locally compact groups..........

N. V. Rao and Ashoke Kumar Roy, Linear isometries of some function

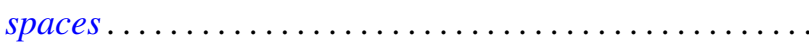

William Francis Reynolds, Blocks and F-class algebras of finite groups

Richard Rochberg, Which linear maps of the disk algebra are multiplicative ...

Gary Sampson, Sharp estimates of convolution transforms in terms of decreasing

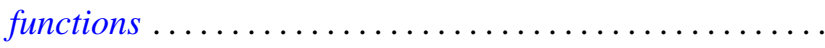

Stephen Scheinberg, Fatou's lemma in normed linear spaces

Ken Shaw, Whittaker constants for entire functions of several complex

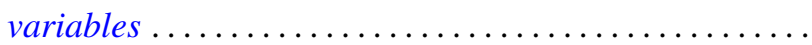

James DeWitt Stein, Two uniform boundedness theorems................ 251

$\mathrm{Li} \mathrm{Pi} \mathrm{Su,} \mathrm{Homomorphisms} \mathrm{of} \mathrm{near-rings} \mathrm{of} \mathrm{continuous} \mathrm{functions} \mathrm{.} \mathrm{.............} 261$

Stephen Willard, Functionally compact spaces, $C$-compact spaces and mappings of minimal Hausdorff spaces....................... 